\title{
Identifying parasitic current pathways in CIGS solar cells by modelling dark $\boldsymbol{J}-\boldsymbol{V}$ response
}

\author{
Benjamin L. Williams ${ }^{1}{ }^{*}$, Sjoerd Smit ${ }^{1}$, Bas J. Kniknie ${ }^{2,4}$, Klaas J. Bakker ${ }^{3,4}$, Wytze Keuning ${ }^{1}$ \\ W. M. M. Kessels ${ }^{1,4}$, Ruud E. I. Schropp ${ }^{1,3,4}$ and M. Creatore ${ }^{1,4 *}$ \\ 1 Department of Applied Physics, Eindhoven University of Technology, P.O. Box 513, 5600 MB Eindhoven, The Netherlands \\ 2 TNO, P.O. Box 6235, 5600 HE Eindhoven, The Netherlands \\ 3 ECN, High Tech Campus, 5656 AE Eindhoven, The Netherlands \\ 4 Solliance, High Tech Campus 21, 5656 AE Eindhoven, The Netherlands
}

\begin{abstract}
The non-uniform presence of shunting defects is a significant cause of poor reproducibility across large-area solar cells, or from batch-to-batch for small area cells, but the most commonly used value for shunt parameterisation (the shunt resistance) fails to identify the cause for shunting. Here, the use of equivalent circuit models to describe dark current-voltage characteristics of $\mathrm{ZnO}: \mathrm{Al} / \mathrm{i}-\mathrm{ZnO} / \mathrm{CdS} / \mathrm{CIGS} / \mathrm{Mo}$ devices in order to understand shunting behaviour is evaluated. Simple models, with a single shunt pathway, were tested but failed to fit experimental data, whereas a more sophisticated model developed here, which includes three shunting pathways, yielded excellent agreement throughout the temperature range of $183-323 \mathrm{~K}$. The temperature dependence of fitting parameters is consistent with known physical models. Activation energies and contact barriers are determined from the model, and extracted diode factors are unique across the voltage range. A case study is presented whereby the model is used to diagnose poor reproducibility for CIGS devices (efficiency $\sim 3-14 \%$ across a $100 \mathrm{~cm}^{2}$ plate). It's shown that lower efficiencies correlated with greater prevalence of Ohmic and non-Ohmic shunt currents, which may form due to pinholes in absorber and buffer layers respectively, whereas the quality of the main junction was constant for all cells (diode factor 1.5-2). Electron microscopy confirmed the presence of $\mathrm{ZnO}: \mathrm{Al} / \mathrm{i}-\mathrm{ZnO} / \mathrm{Mo}$ and $\mathrm{ZnO}: \mathrm{Al} / \mathrm{CIGS} / \mathrm{Mo}$ regions, supporting the multi-shunt pathway scheme disclosed by modelling. While the model is tested with CIGS cells here, this general model is a powerful diagnostic tool for process development for any type of thin-film device. Copyright () 2015 John Wiley \& Sons, Ltd.
\end{abstract}

\section{KEYWORDS}

dark current-voltage; CIGS; shunt resistance; modelling

*Correspondence

Williams Benjamin and Mariadriana Creatore, Applied Physics, Eindhoven University of Technology, The Netherlands.

E-mail: b.I.williams@tue.nl; m.creatore@tue.nl

Received 7 July 2014; Revised 16 September 2014; Accepted 27 October 2014

\section{INTRODUCTION}

Recently there has been significant progress in some of the leading thin-film photovoltaic technologies, with copper indium gallium diselenide (CIGS) and CdTe devices now boasting world record efficiencies of $21.7 \%$ and $21.0 \%$, respectively [1]. However, reproducibility is as much sought-after as peak efficiency, so that quality assurance and baseline efficiency can be maintained. Both at industrial- and lab-scale, large spreads in efficiency are often observed for thin-film technologies, either between multiple batches, or within single batches. To some extent, this is attributed to non-uniformities of the intrinsic material properties of individual device layers, which may arise due to spatial variations during deposition and/or postgrowth processing stages. To a larger extent, however, it is attributed to the non-uniform occurrence of shunting defects, i.e. short-circuit pathways that enable current to bypass the main junction. High leakage (or shunt) currents are deleterious to fill factor $(F F)$, open-circuit voltage $\left(V_{O C}\right)$, and in extreme cases, to short-circuit current $\left(J_{S C}\right)$. There are a number of possible causes for shunting: micro-scale pinholes in individual layers of the device [2]; segregation of conductive chemical elements towards 
grain boundaries and the subsequent formation of low resistance pathways [3]; or a high density of mid-gap defects facilitating tunneling currents [4]. However, the ubiquitous method of quantifying shunting-i.e. the measurement of a single parameter, the shunt resistance $\left(R_{S H}\right)$ - does not provide understanding as to the type of shunt present. Alternatively, one can construct an equivalent circuit model, consisting of an ideal diode component in parallel with a shunting pathway, in order to describe the electrical behaviour of a device, and then assess the model's validity by fitting it to experimental current density-voltage $(J-V)$ data. In this way, different charge transport physics for the shunting pathway can be tested in terms of its ability to replicate the data. This shunting pathway is predominantly considered to be Ohmic [5] and may therefore account for pinholes in the absorber layer [6] which enable short-circuits between the conductive window layers and the back contact. However, Dongaonkar [7] et al. recently demonstrated that shunting in CIGS, thin film silicon, and organic solar cells can be non-Ohmic in nature. In other cases, it is highly plausible that Ohmic, non-Ohmic, and even tunnelling currents exist simultaneously, and so it is necessary to design an equivalent model that accounts for all these components, so that their presence can be identified.

In this work, the necessity of using such a general model (explained in Section 2) as opposed to simpler models to describe dark $J-V$ characteristics of CIGS solar cells is evaluated by fitting various models to experimental data of $\mathrm{ZnO}: \mathrm{Al} / \mathrm{i}-\mathrm{ZnO} / \mathrm{CdS} / \mathrm{CIGS} / \mathrm{Mo}$ devices. Up to eight fitting parameters are used in the general model, and so to assess the validity of including each of the equivalent circuit components, fitting of experimental data over a large temperature range (183-323 K) is also demonstrated, and the temperature dependence of the free parameters is compared to their expected behaviour. Finally, a case study is presented whereby the model is applied to a set of CIGS solar cells of wide-ranging efficiency from the same processing batch in order to identify the cause of poor cell-to-cell reproducibility. Electron microscopy was also used to provide supporting information, i.e. to identify the possible causes of the leakage pathways in this case study to support the multi-shunt model. Ultimately we propose that this electrical modelling methodology can provide rapid diagnosis of low, or inconsistent, efficiencies. In this way process development for thin-film solar cells may be accelerated.

\section{DESCRIPTION OF MODEL}

First, the physical causes of various current components that may be included in an equivalent circuit model are described. Figure 1 shows a schematic depicting of possible current paths through a $\mathrm{ZnO}: \mathrm{Al} / \mathrm{i}-\mathrm{ZnO} / \mathrm{CdS} / \mathrm{CIGS} / \mathrm{Mo}$ solar cell. Note that a metal (e.g. $\mathrm{Al} / \mathrm{Ni}, \mathrm{Cu}$ or $\mathrm{Au}$ ) grid on top of the $\mathrm{ZnO}: \mathrm{Al}$ (or indium tin oxide) front contact is also used in many lab-scale devices to enhance carrier collection.

In coincidence with the diffusion/recombination dominated diode current of the unperturbed junction, there may be: (i) Ohmic shunts due to pinholes in the absorber layer, or via low-resistance grain boundaries, (ii) spacecharge limited currents (SCLC) due to the presence of metal/semiconductor/metal-like regions, which may form upon metal diffusion from the front contact (or metal grid) and through the buffer layers, or due to pinholes in the buffer layers and the subsequent localised absence of the CdS/CIGS built-in field; and (iii) tunnelling currents via high densities of mid-gap defect states in the depletion region of the $p-n$ junction. Whilst it is not expected that all defects are present in all devices, by allowing for their inclusion in a general model, one can test for their presence by fitting the model to experimental data. Characteristic $J-V$ behaviours that may signify the presence of such defects are now discussed.

\subsection{Reverse-bias behaviour}

Dark current-voltage $(J-V)$ curves are dominated by shunt currents in reverse bias (see Figure 2a). The traditional Ohmic model for shunt current predicts a linear response, and so any deviation from this implies the presence of non-Ohmic shunt currents. For thin-film solar cells, SCLC is the most likely candidate to describe non-Ohmic shunts [7]. Non-linear dark current in reverse bias may also be an effect of diode breakdown, but this rarely occurs in the voltage range that is typically used for solar cell measurements (up to $-1 \mathrm{~V}$ ).

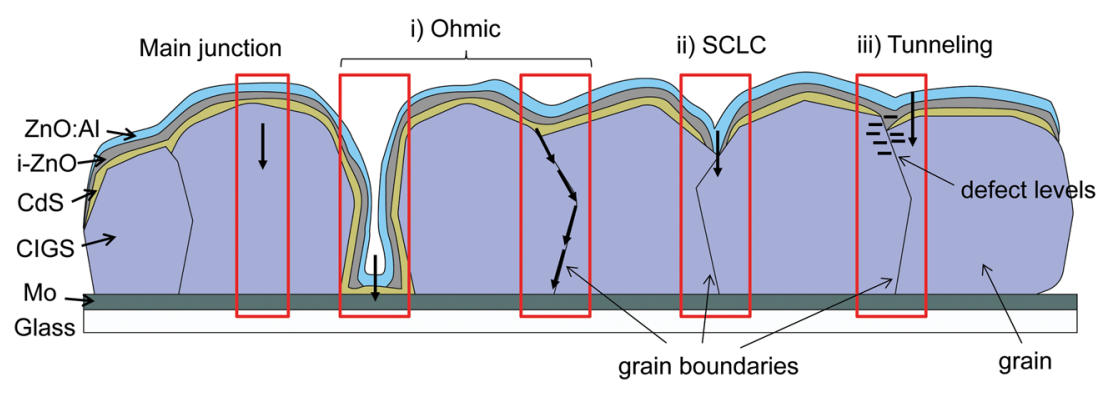

Figure 1. Possible shunt pathways in a $\mathrm{ZnO}: \mathrm{Al} / \mathrm{i}-\mathrm{ZnO} / \mathrm{CdS} / \mathrm{CIGS} / \mathrm{Mo}$ solar cell. SCLC refers to space-charge limited current. 

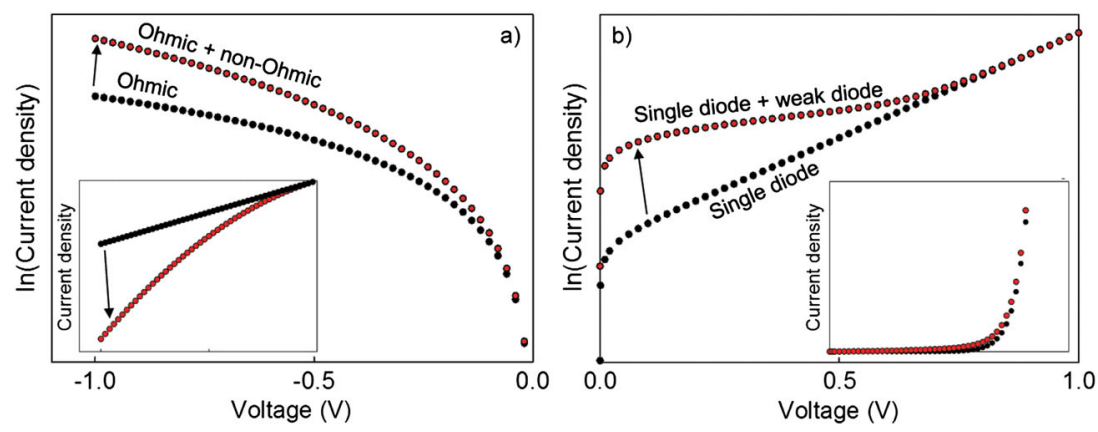

Figure 2. Examples of the effect of non-ideal current pathways on dark $J-V$ curves. Data shown on both semi-log and linear (inset) plots. a) Reverse bias_-any shift from a linear Ohmic shunt current should indicate the presence of an additional shunt pathway. b) Forward bias_kinks in the forward bias semi-log $I-V$ plot may indicate the presence of weak diode regions.

\subsection{Forward-bias behaviour}

In forward bias, one may expect a response that is dominated by the diode-like behaviour of the main junction (see the black data points in Figure 2b). In this case the slope of $\ln (J)-V$, and therefore the diode ideality factor $(n)$, is constant with $V$. However, it is common to observe shoulders in $\ln (J)-V$ plots (red data points in Figure $2 b$ ), and this can be explained either by: (i) the classic twodiode model-this assumes recombination dominated transport $(n=2)$ at low forward bias and diffusion dominated transport $(n=1)$ at high forward bias; or (ii) an alternative two diode model which consists of a main junction diode $(n=1-2)$ and a tunnelling component which has a similar exponential dependence as a diode. Note that this behaviour is not clearly identifiable when $J$ is plotted on a linear scale (Figure 2b inset).

\subsection{Complete general model}

This general model therefore consists of four parallel current pathways: (i) the main junction diode; (ii) a second exponential term (which may describe either a weak diode, i.e. with a higher saturation current than the main junction or with recombination-dominated current transport-or multi-step tunnelling); (iii) an Ohmic leakage current; and (iv) a non-Ohmic (SCLC) leakage current. These current components can be expressed as follows:

$$
\begin{gathered}
J_{\text {main junction }}=J_{01}\left(\mathrm{e}^{A_{1} V}-1\right) \\
J_{\text {weak diode/tunneling }}=J_{02}\left(\mathrm{e}^{A_{2} V}-1\right) \\
J_{\text {Ohmic shunt }}=V / R_{S H} \\
J_{S C L C}=k V^{m}
\end{gathered}
$$

where $J_{01}$ and $J_{02}$ are the saturation currents of the main junction and second exponential term, respectively, and $A_{1}$ and $A_{2}$ are their exponents, $k$ is the coefficient of the SCLC component (related to the length and conductivity of the current path), and $m$ is its power factor (which should be $\sim 2$ as defined in Ref. [8] in the case whereby shallow traps facilitate the current, or $>2$ if a high density of deep traps are present). These components are connected with a single series resistance $\left(R_{S}\right)$ component, giving the full expression for the dark current $\left(J_{D}\right)$ as:

$$
\begin{aligned}
J_{D}= & J_{01}\left(\mathrm{e}^{A_{1}\left(V-J_{D} R_{S}\right)}-1\right)+J_{02}\left(\mathrm{e}^{A_{2}\left(V-J_{D} R_{S}\right)}-1\right) \\
& +\left(V-J_{D} R_{S}\right) / R_{S H}+k\left(V-J_{D} R_{S}\right)^{m}
\end{aligned}
$$

For the first two components, diode ideality factors may be calculated according to the relationship, $n=q / A k T$, where $q$ is the electron charge, $k$ is Boltzmann's constant, and $T$ is the temperature. However in the case whereby (2) describes a tunnelling current, $n$ is not a physically valid parameter, and we maintain the use of $A$ as the exponent parameter.

\section{EXPERIMENTAL DETAILS}

$\mathrm{ZnO}: \mathrm{Al} / \mathrm{i}-\mathrm{ZnO} / \mathrm{CdS} / \mathrm{CIGS} / \mathrm{Mo}$ solar cells were fabricated on $10 \times 10 \mathrm{~cm}$ plates of soda-lime glass. The $(2 \mu \mathrm{m}$ thick $)$ CIGS layer was generated by co-evaporation, the CdS $(50 \mathrm{~nm})$ by chemical bath deposition, the Mo $(400 \mathrm{~nm})$ and $\mathrm{i}-\mathrm{ZnO}(50 \mathrm{~nm})$ by $\mathrm{RF}$ sputtering and the $\mathrm{ZnO}$ :Al either by RF sputtering $(430 \mathrm{~nm})$ or chemical vapour deposition $(700 \mathrm{~nm})$. For contacting, $\mathrm{Cu}$ finger contacts were deposited onto the $\mathrm{ZnO}: \mathrm{Al}$ by thermal evaporation, and individual cells $(5 \mathrm{~mm} \times 10 \mathrm{~mm})$ were defined by scribing. Light current density-voltage $(J-V)$ data were recorded under Air Mass (AM) 1.5 conditions at $25^{\circ} \mathrm{C}$ (to determine efficiency), and dark $J-V$ data (for model fitting) were recorded at temperatures in the range of 183-323 K: Samples were held in a Linkam THMS600 stage and cooled with a continuous flow of liquid nitrogen using a Linkam LNP95 system. Fitting of dark $J-V$ curves with Equation 5 was implemented using Mathematica. Planview scanning electron microscope (SEM) images were acquired in secondary-electron mode in a FEI Nova 600i NanoLab dual-beam system. The same system was used to generate cross-sectional SEM images: a focussed beam of $\mathrm{Ga}$ ions were used to mill a trench in the sample and 
to polish a cross section. This was then also imaged in secondary electron mode.

\section{RESULTS AND DISCUSSION}

\subsection{Validation of model and comparison with simpler models}

First, the dark $J-V$ curve of a cell having an efficiency of $11.2 \%$ was selected for fitting with Equation 5. This cell had an open-circuit voltage $\left(V_{O C}\right)$ of $625 \mathrm{mV}$, fill factor $(F F)$ of $55.3 \%$, and short-circuit current density $\left(J_{S C}\right)$ of $32.5 \mathrm{~mA} \cdot \mathrm{cm}^{-2}$. Simpler variations of the model (i.e. whereby certain terms are omitted) were also tested, in terms of their ability to generate a good fit.

Figure 3 shows the measured dark curve for the $11.2 \%$ reference cell, and the best obtained fit for five variations of the model. Plots of $\mathrm{d}(\ln J) / \mathrm{d} V-V$ are also included, with the corresponding modelled curves, to help elucidate the quality of the fit. Comments are now made for each model (schematics included in insets in Figures 3a-e, respectively) regarding their level of agreement with the experimental data. Figure $3 a$ (single diode with Ohmic shunt): In reverse bias, experiment and model disagree, and the complexity of the forward bias region is not reproduced. Figure $3 \mathrm{~b}$ (double diode with Ohmic shunt): In reverse bias the model fails, but a good fit is obtained in forward bias. Figure $3 \mathrm{c}$ (single diode with Ohmic and non-Ohmic shunts): In reverse bias there is good agreement, but the complexity of the forward bias region is not reproduced. Figure 3d (double diode with non-Ohmic shunt): A good fit cannot be obtained in low-reverse and low-forward biases. Figure $3 e$ (double diode with Ohmic and non-Ohmic shunts, i.e. Equation 5): A good fit is obtained throughout the bias range. It is clear that for this device, two exponential terms are necessary in order to provide a good fit in forward bias, and both Ohmic and non-Ohmic shunt pathways are necessary in reverse bias (although note that the entire voltage range is fit simultaneously with Equation 5).

The fitting parameters for the $11.2 \%$ reference cell are given in Table I. Since a large number of fitting parameters is used, it is important to assess the validity of their values: The ideality factor of the main junction, $n_{1}$, is 1.4 $\left(A_{1}=26.9\right)$, i.e. within the allowed limits of a diode in which diffusion $(n \sim 1)$ or recombination $(n \sim 2)$ dominates transport. Whilst $n$-values exceeding 2 are often reported when they are determined directly from the gradient of $1 n$ $(J)-V$ curves [9-12], this should not be the case when using this methodology since the main junction component is isolated from any influence by the shunt components and series resistance. However, the $n$ value of the secondary exponential term is $5.9\left(A_{2}=6.5 \mathrm{~V}^{-1}\right)$ which may imply that the term describes a tunnelling current (this is further investigated in Section 4.2). The reverse saturation current of the second exponential term, $J_{O 2}\left(7.9 \times 10^{-2} \mathrm{~mA}\right)$ is significantly higher than that of the main junction $\left(J_{O I}=3.2 \times 10^{-7} \mathrm{~mA}\right)$, and so can be considered to be a non-negligible contribution to the total current in reverse bias. The power factor of the SCLC term, $m$, is 2.4 , and this is consistent with SCLC transport via a semiconductor with a significant trap density within the band gap. $R_{S H}$ $\left(270 \Omega \cdot \mathrm{cm}^{2}\right)$ and $R_{S}\left(1.2 \Omega \cdot \mathrm{cm}^{2}\right)$ are of realistic magnitude for thin-film solar cells, but note that this extracted $R_{S H}$ value is higher than that which would be determined simply from taking the $J-V$ gradient at $0 \mathrm{~V}\left(176 \Omega \cdot \mathrm{cm}^{2}\right)$.

With regards the uniqueness of the fit, note that the same parameter set was achieved when using a wide range of initial conditions for all the fitting parameters. A further test of the robustness of the model is to hold one parameter fixed (allowing the remaining parameters free) during fitting and to demonstrate that the value of the fixed parameter that yields the best fit (i.e. minimised chi-squared) is the same as that extracted when all parameters are free. Using $A_{l}$ and $k$ as examples of this fixed parameter, we demonstrate in Figure S1 in the supporting information that this was indeed the case.

\subsection{Temperature dependence of fitting parameters}

Here, to evaluate the validity of including each of the components in the equivalent circuit model, the temperature dependence of the fitting parameters is assessed. Figure 4 shows dark $J-V$ curves of a CIGS cell $(\eta=10.0 \%)$ taken at various temperatures (183-323 K) each fit with Equation 5, and it is seen that excellent agreement between experimental and modelled data was achieved throughout this range. Note that this is a different cell than in Section 4.1 to verify that multiple cells may be fit by the model (see Section 4.3 and the supporting information for further examples). A discontinuity in the data is seen between $\mathrm{T}=223 \mathrm{~K}$ and $\mathrm{T}=233 \mathrm{~K}$; this is most likely related to defreezing of metastable states, which are well known in CIGS devices.

Figures 5a-f show the temperature dependences of the extracted fitting parameters. Importantly, all fitting parameters follow their expected temperature dependence-these are summarised in Table II, and discussed below. Moreover, some important device constants, such as activation energies and contact-barrier heights, can be determined from these relationships, and this is also discussed.

For the main junction, transport is assumed to be dominated by diffusion/recombination, and this is supported by the observation that $A_{l}$ increases as $T$ decreases (Figure 5a) $[9,10,13]$. This corresponds with a gradual decrease of $n_{1}$ from 1.5 at $323 \mathrm{~K}$ to 1.1 at $183 \mathrm{~K}$ (not shown), and hence a transition to a diffusion-dominated regime. Moreover, $J_{O I}$ followed the relationship $\ln J_{0} \propto-1 / T$ (Figure $5 \mathrm{~b}$ ), as is expected according to the following expression for $J_{0}$ in the diffusion/recombination regime [13];

$$
J_{0}=J_{00} e^{-\Delta E / n k T}
$$

where $J_{00}$ is a constant. From the slope of $\ln J_{0} \propto-1 / T$, the activation energy $(\Delta E)$ was determined to be $1.5 \pm 0.2 \mathrm{eV}$. 

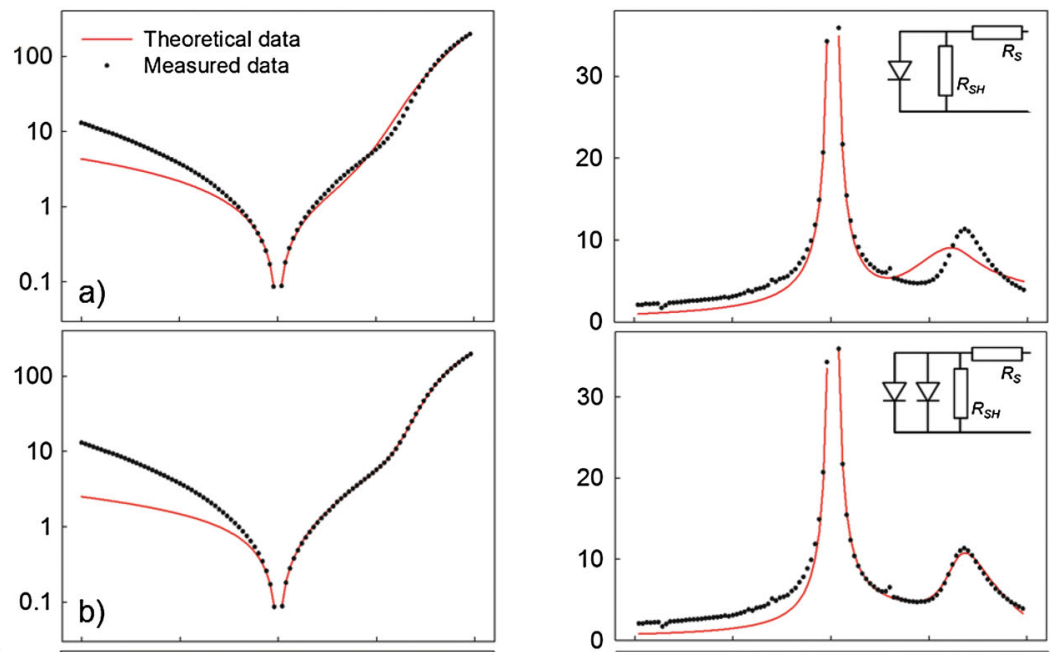

0.1 b)
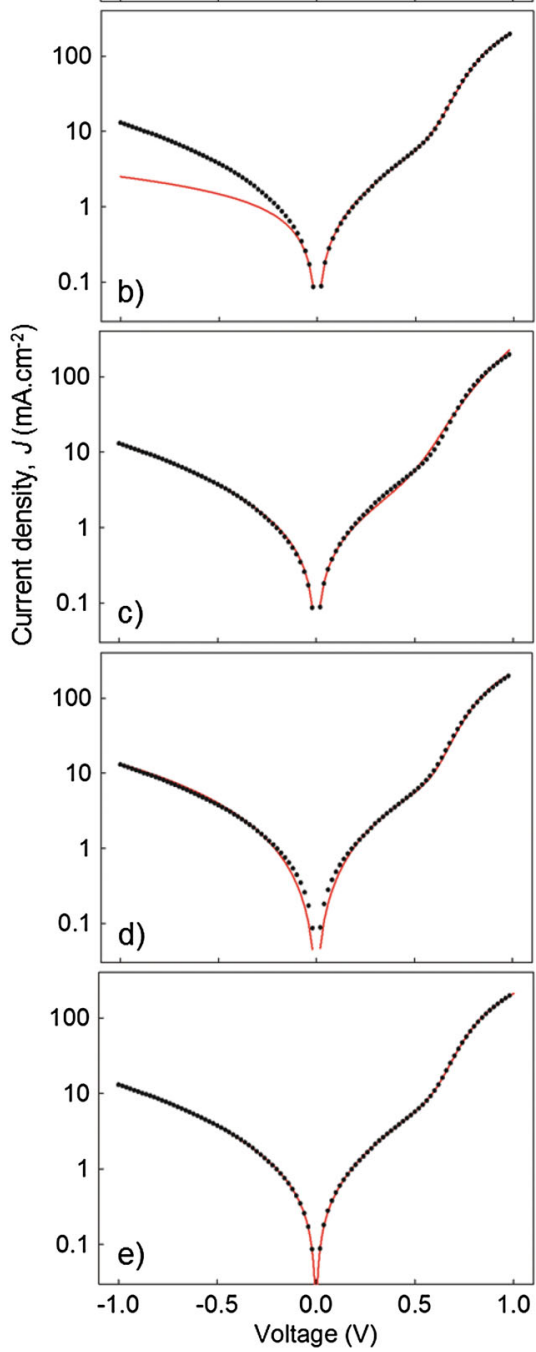
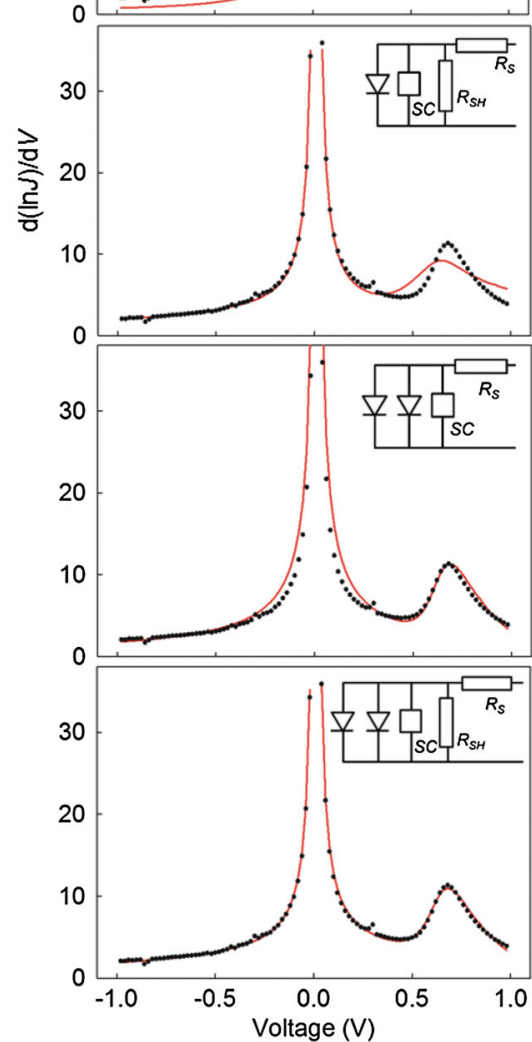

Figure 3. Measured dark $J-V$ and $d(\operatorname{In} J) / d V-V$ curves of a reference $C I G S$ solar cell $(\eta=11.2 \%)$ fit with various electric circuit models: a) single diode with Ohmic shunt, b) double diode with Ohmic shunt, c) single diode with Ohmic and non-Ohmic shunts, d) double diode with non-Ohmic shunt, and e) double diode with Ohmic and non-Ohmic shunts.

This value is slightly higher than the band gap of the CIGS absorber used here $\left(E_{G} \sim 1.2 \mathrm{eV}\right)$, but nevertheless for $\Delta E \sim E_{G}$ one infers that recombination processes in the main junction component are dominated by ShockleyRead-Hall statistics. Possible reasons for measuring an activation energy larger than the CIGS band gap are: (i) the temperature dependence of the band gap (the band gap of the material is expected to be up to $0.05 \mathrm{eV}$ larger at $183 \mathrm{~K}$ than at room temperature and the value is determined from the entire temperature range); and (ii) band- gap enlargement close to the interface with CdS due to either S-diffusion into the CIGS or Cu-depletion from the CIGS. The observations that $A_{2}$ is relatively independent of temperature (Figure 5a) and $J_{02}$ follows the relationship $\ln J_{0} \propto T$ (Figure $5 \mathrm{c}$ ) are consistent with the second exponential term describing a current facilitated by multi-step tunnelling [14]. Given that $J_{02}$ is orders of magnitude higher than $J_{O I}$, this 'tunnelling' component must be considered to be a non-negligible contribution to the leakage current, particular in the forward bias. 
Table I. Parameters extracted from fitting dark $J-V$ data of a CIGS device with $\eta=11.2 \%$.

\begin{tabular}{ll}
\hline$J_{O 1}$ & $3.2 \times 10^{-7} \mathrm{~mA} \cdot \mathrm{cm}^{-2}$ \\
$A_{1}$ & $26.9 \mathrm{~V}^{-1}\left(n_{1}=1.4\right)$ \\
$J_{O 2}$ & $7.9 \times 10^{-2} \mathrm{~mA} \cdot \mathrm{cm}^{-2}$ \\
$A_{2}$ & $6.5 \mathrm{~V}^{-1}$ \\
$k$ & $9.6 \mathrm{AN} \mathrm{N}^{\mathrm{m}}$ \\
$m$ & 2.4 \\
$R_{S H}$ & $270 \Omega \mathrm{cm}^{2}$ \\
$R_{S}$ & $1.2 \Omega . \mathrm{cm}^{2}$ \\
\hline
\end{tabular}
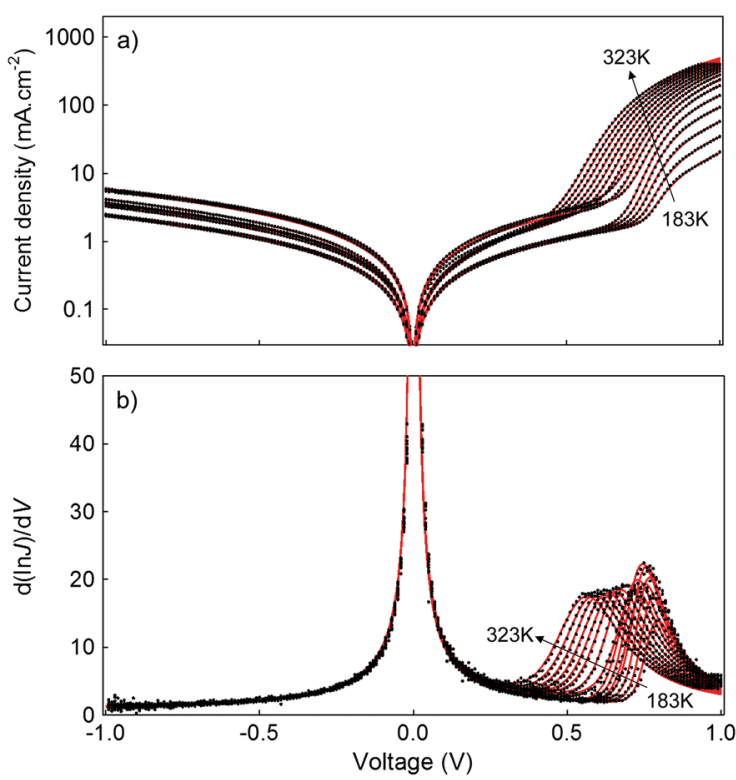

Figure 4. Experimental (black circles) dark a) $J-V$ curves and b) $\mathrm{d}(\operatorname{In} J) / \mathrm{d} V$ curves of a device with $\eta=10.0 \%$, each fit with Equation 5 (solid red lines) at a range of temperatures (from 183 to $323 \mathrm{~K}$ in steps of $10 \mathrm{~K}$ ). The same parameter sets were used for the fits in $a$ and $b$.

For the SCLC component, $m$ is inversely proportional to $T$ (Figure $5 \mathrm{~d}$ ), which is consistent with the relationship defined by Rose [8], i.e. $m=\left(T_{c} / T\right)+1$, in which $T_{C}$ defines the steepness of the energy distribution of the traps (from the conduction band edge) which facilitate current transport: A high value correlates to a slow variation of trap distribution. At temperatures above $T_{C}$, the SCLC current is governed by shallow traps. Here, $T_{C}$ was determined to be $453 \mathrm{~K}$. To put this into context, it is higher than that previously observed in CdTe thin films (380 K) [15], but an order of magnitude lower than in organic solar cells [16]. Note that relative to other cells presented here, the $m$-value at room temperature (2.1) is different to that determined for the $11.2 \%$ device $(m=2.4)$ and indeed to other cells measured here (see Section 4.3), pointing to a difference in the trap distribution from cell-to-cell. The values of $m$ and $T_{C}$ are influenced both by bulk trap states and interface traps, so since device processing was the same for all cells, the different $m$ values may be accounted for by the non- uniform prevalence of structural defects that are described in Section 4.4.

It has been previously demonstrated by Marsal [17] et al. that the coefficient, $k$, of the SCLC increases with temperature in a-Si/c-Si heterojunction devices, and the same behaviour was observed here (Figure 5f). Since $k$ is defined by the physical length of the SCLC region (i.e. film thickness), its conductivity, and the trapping behaviour, this temperature dependence may either be related to thermally activated de-trapping of carriers, or simply a change of conductivity of the SCLC region with temperature.

$R_{S}$ follows the exponential dependence proposed by Bätzner [18] et al. for barrier-dominated series resistances (Figure 5e). From this, via the method of Bätzner, the height of the barrier, $\varnothing_{B}$, formed within the device was determined to be $0.16 \mathrm{eV}$, which is relatively low and so indicates that performance is not limited by the barrier: only barrier heights exceeding $0.3 \mathrm{eV}$ are considered to be detrimental to device efficiency [19] This barrier may arise from a Schottky contact at the Mo/CIGS interface or from a conduction band offset at the CdS/CIGS interface. The latter is usually accompanied with a 'crossover' of the light and dark curves at high forward bias [20], and since this wasn't seen here, we speculate that the source of the barrier is more likely to be at the back contact. $R_{S H}$ is considered to quantify a purely Ohmic shunt in this model, and so one would expect an approximately linear temperature-dependence: Indeed, in the $T$-range of $232-323 \mathrm{~K}, R_{S H}$ increased linearly from 200 to $600 \Omega \cdot \mathrm{cm}^{2}$ (not shown), but there was some scatter at $T<232 \mathrm{~K}$, which may be related to the freezing/defreezing of metastable states, as mentioned above.

The consistency between the $T$-dependences of the fitting parameters and the respective theoretical models and/or literature observations is further validation of the proposed $J-V$ model. Furthermore, a number of device constants, such as $\Delta E$ and $\varnothing_{B}$, can be extracted from this data. Indeed, since this methodology allows isolation of the various current components, it should serve to be a more reliable procedure for device parameter extraction than common manual methods. For instance, $n$ and $J_{O}$ are often determined directly from the gradient and intercept of $\ln (J)-V$ plots, respectively, but the common observation is that the manually extracted values are voltage dependent [12], owing to the non-constant $\ln (J)-V$ slope. Manual extraction also results in strongly $T$-dependent $n$-values $[9,10]$, attributed to transitions between different current transport regimes. Here, by isolating the main junction from the effect of $R_{S}, R_{S H}$ and other non-idealities, a single, voltage-independent $n$-value is extracted. Moreover, its temperature dependence is minimal and within the allowed limits of the ideality factor $(1 \leq n \leq 2)$.

\subsection{Case study of CIGS poor reproducibility}

In this section, the application of the model to a case study is presented in order to demonstrate its effectiveness for diagnosis of inconsistent device performance. From the 

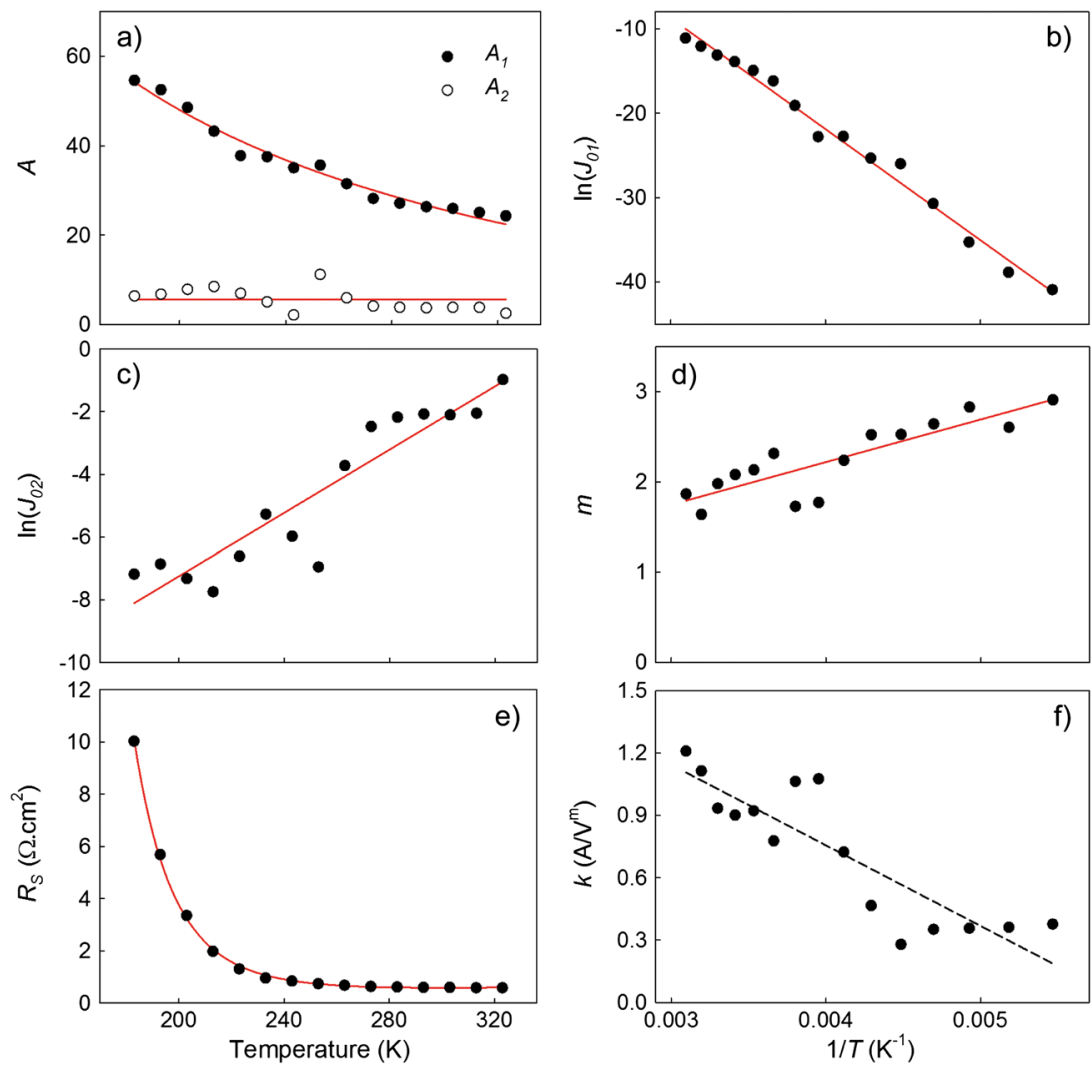

Figure 5. Temperature dependence of fitting parameters; a) $A_{1}$ and $\left.\left.A_{2}, \mathrm{~b}\right) \ln \left(J_{01}\right), c\right) \ln \left(J_{02}\right)$, d $) m$, e) $R_{s}$, and f) $k$. (a), (c), and (e) are plotted against $T$, and (b), (d), and (f) are plotted against $1 / T$. The red curves and lines correspond to the models described in Table II, and the line in (f) is added as a guide to the eye.

Table II. Expected temperature dependences for fitting parameters. Note the dependences for $J_{01}$ and $A_{1}$ are assuming recombination/diffusion dominated transport, and those for $J_{02}$ and $A_{2}$ are assuming tunnelling dominated transport. $J_{00}, B, T_{C}$, and $C$ are constants, $\Delta E$ is the activation energy of the recombination/diffusion process, and $\varnothing_{B}$ is the back contact barrier height.

\begin{tabular}{lll}
\hline Parameter & Expected $T$ dependence & Ref. \\
\hline$J_{O 1}$ & $J_{O}=o e^{-\Delta E / n k T} \equiv \ln J_{O^{\alpha}-1 / T}$ & {$[9,10,13]$} \\
$A_{1}$ & $A=q / n k T$ & {$[9,10,13]$} \\
$J_{O 2}$ & $J_{O}=J_{O O} e^{B T} \equiv \ln J_{O^{\alpha} T}$ & {$[14]$} \\
$A_{2}$ & no $T$-dependence & {$[14]$} \\
$m$ & $m=\left(T_{C} / T\right)+1 \equiv m \propto 1 / T$ & {$[8]$} \\
$k$ & increase with $T$ & {$[17]$} \\
$R_{S}$ & $R_{S}=\left(C / T^{2}\right) e^{\sigma_{B / k T}}$ & {$[18]$} \\
$R_{S H}$ & $R_{S H^{\alpha} T}$ & - \\
\hline
\end{tabular}

same $10 \mathrm{~cm} \times 10 \mathrm{~cm}$ plate the light and dark $J-V$ curves of 18 cells were measured. From the light measurements, it was seen that there was a wide spread in efficiency (3.5-13.8\%), and this was predominantly linked to the spread in $F F$ (from 29 to $69 \%$ ), since $J_{S C}\left(30-32 \mathrm{~mA} \cdot \mathrm{cm}^{2}\right.$ ) and $V_{O C}(518-623 \mathrm{mV})$ were comparatively consistent. Indeed, a simple diagnosis would be that low efficiencies are caused by excessive shunting. However, the use of the multi-component model allows more in-depth analysis, and so each of the dark $J-V$ curves was fit with Equation 5. Examples of some of the fits for both low and high efficiency cells are shown in the supporting information (Figure S2).

Following parameter extraction, it could be seen that the lower efficiencies corresponded to both a decrease of $R_{S H}$ (Figure 6a) and an increase of $k$ (Figure 6b), as well as (to a lesser extent) an increase in $J_{O 2}$ (not shown). In contrast, there was no clear correlation between efficiency $(\eta)$ and $n_{l}$, which serves as an indicator of the quality of the main junction (see Figure 6c); for both low and high efficiency devices, $n_{l}$ was in the range 1.5-2. Similarly, no clear correlation between efficiency and $m$ (in the range 2.2-3.5), and efficiency and $J_{O I}$ (typically of the order $10^{-6} \mathrm{~mA} \cdot \mathrm{cm}^{-2}$ ) was seen. $R_{S}$ was actually slightly lower for the lower efficiency cells (in the range $0.6-1.2 \Omega \cdot \mathrm{cm}$ for the data set, see Figure 6d). Hence, the large spread in cell efficiency is entirely attributed to the non-uniform prevalence of multiple shunt pathways, as opposed to significant chemical inhomogeneities. The device parameter most affected was the $F F$, but since $V_{O C}$ is also affected when shunting is significantly high, a notably large spread in $V_{O C}$ was also seen. 

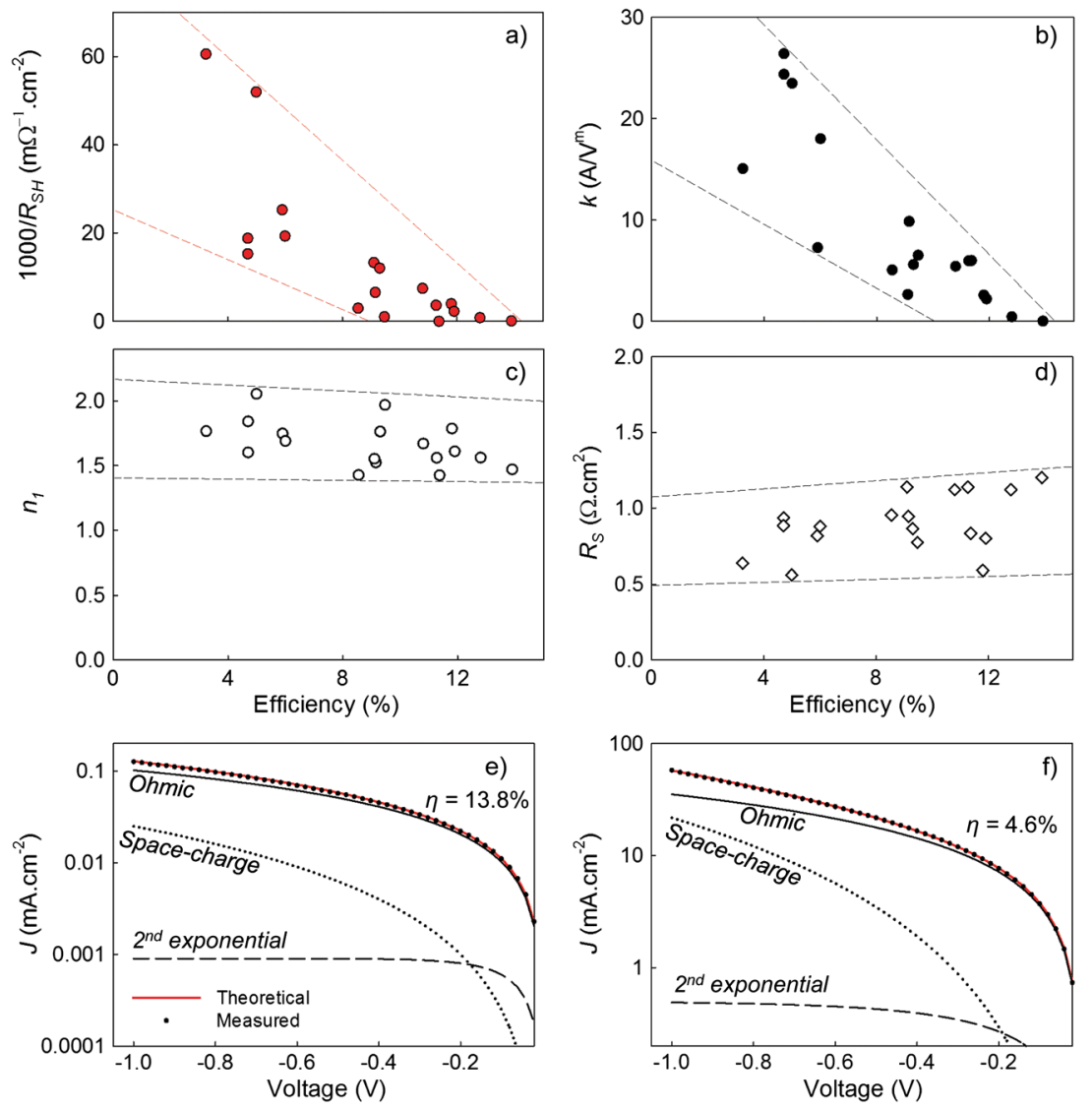

Figure 6. Extracted values (from fitting dark $J-V$ curves) for a) $1000 / R_{S H}$, b) $\left.k, c\right) n_{1}$, and d) $R_{S}$ plotted vs. cell efficiency. e) and f) Reverse bias regions of the dark $J-V$ response of high (13.8\%) and low (4.6\%) efficiency devices respectively, fit with Equation 5 and deconvoluted to show the contributions of the individual circuit elements. Lines in (a)-(d) are added as guides to the eye.

Figure 6e shows a deconvolution of the reverse bias region for a $13.8 \%$ efficient device, demonstrating that the Ohmic component is most significant in this case $(\sim 80 \%$ of the total shunt current at $-1 \mathrm{~V})$. The same plot for a $4.6 \%$ efficient device (Figure $6 f$ ) shows that, in comparison to the $13.8 \%$ device, all three shunt components are significantly higher (note the difference in scale on the $y$-axis), and the relative contribution of the SCLC is greater (here, the Ohmic component accounts for $\sim 60 \%$ of the total shunt current at $-1 \mathrm{~V}$ ).

\subsection{Physical origin of shunt pathways}

Scanning electron microscopy (SEM) analysis was carried out in order to identify the presence of structural defects which can account for the multiple shunt pathways elucidated by the model. Figure 7 a shows a macro-scale 'crater' found in one of the cells, which is representative of similar features found in most of the cells examined. Upon examining $\sim 40$ cells from three different $10 \times 10 \mathrm{~cm}$ plates, the average density of these defects was $\sim 1$ per $0.5 \mathrm{~cm}^{2}$ cell, although in some cells up to 5 were present. These craters are created following the formation of secondary-phase $\mathrm{CuSe}_{\mathrm{x}}$ particles during CIGS co- evaporation, and their subsequent removal during window layer deposition (see Ref. 21). A focussed ion beam (FIB)-SEM cross section of such a feature (Figure 7b) shows that whilst 'outside' the crater (towards the left of the image) the full unperturbed $\mathrm{ZnO}: \mathrm{Al} / \mathrm{i}-\mathrm{ZnO} / \mathrm{CdS} /$ CIGS/Mo stack can be seen with a CIGS thickness of $\sim 2 \mu \mathrm{m}$, 'inside' the crater (towards the right of the image) the CIGS layer is significantly thinner. At higher magnifications, the presence of $\mathrm{ZnO}: \mathrm{Al} / \mathrm{i}-\mathrm{ZnO} / \mathrm{CdS} / \mathrm{Mo}$ (Figure 7c) and $\mathrm{ZnO}: \mathrm{Al} / \mathrm{CIGS} / \mathrm{Mo}$ (Figure 7d) regions, which may be responsible for Ohmic and non-Ohmic shunt pathways respectively, can be seen. The presence and removal of the $\mathrm{CuSe}_{\mathrm{x}}$ particles evidently disrupt both absorber- and window-layer deposition to enable localised incorrect stacking sequences.

Whilst defects of this type may be exceptional, and somewhat particular to this process, it is important to highlight the consistency between (i) the presence of multiple shunt components in the electrical model and (ii) the observation of different forms of shunt pathways in the micrographs. We therefore propose that our methodology of fitting dark $J-V$ data with a multi-component equivalent circuit model can be used to rapidly diagnose the cause of shunting in CIGS solar cells. In contrast, it may not be 

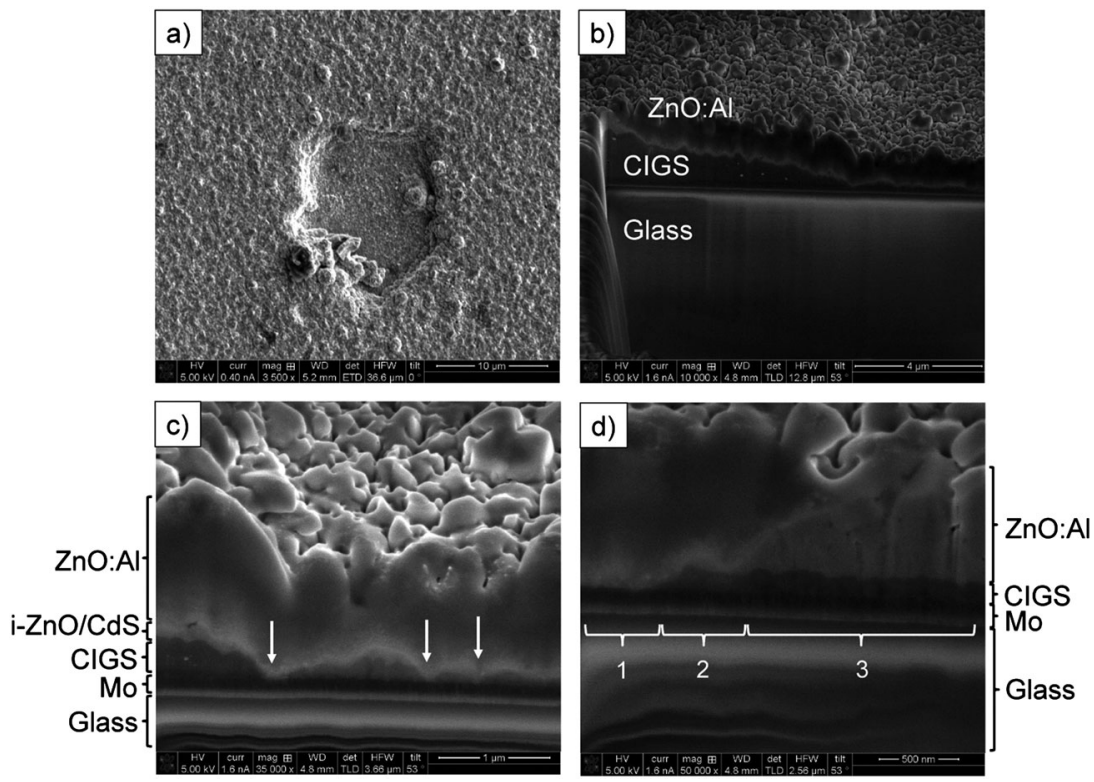

Figure 7. SEM images of a typical crater-like defect observed in the devices: a) Plan-view of a 10- $\mu$ m-wide crater, b) FIB-milled cross section which shows that inside the crater (right) the CIGS absorber layer is significantly thinner than outside (left). Higher magnification images show regions having incorrect stacking sequences as a result of the crater formation: c) ZnO:Al/i-ZnO/CdS/Mo (indicated by the arrows), and d) ZnO:Al/CIGS/Mo (indicated by region 3-note that region 1 consists of $\mathrm{ZnO}: \mathrm{Al} / \mathrm{i}-\mathrm{ZnO} / \mathrm{CdS} / \mathrm{Mo}$ and region 2 of $\mathrm{ZnO}: \mathrm{Al} / \mathrm{i}-\mathrm{ZnO} / \mathrm{CdS} / \mathrm{CIGS} / \mathrm{Mo})$.

practical to frequently use advanced microscopy techniques such as FIB milling/imaging for such diagnosis. Moreover, smaller scale defects (e.g. CdS pinholes caused by film densification) may not be regularly identifiable with SEM. Further work will focus on strengthening the link between specific structural defects and the transport mechanisms of the resultant shunt pathways.

\section{CONCLUSIONS}

An equivalent circuit model has been developed to describe the dark $J-V$ characteristics in CIGS solar cells. Particular focus is paid towards the understanding of shunting behaviour in the cells. The model allows for the presence of three types of shunting pathway (an Ohmic current, a SCLC, and a tunnelling current). Excellent agreement between the model and experimental data was apparent throughout a temperature range of $183-323 \mathrm{~K}$, whereas simpler models, i.e. containing fewer shunt pathways, failed to accurately represent the data. The temperature dependence of all fitting parameters was consistent with the physical models of the individual components. Moreover, the presence of multiple shunt pathways was confirmed by SEM analysis. The model may be used for rapid diagnosis of low, or inconsistent, device efficiencies: In a case study presented here, a large spread in CIGS cell efficiency across a single batch plate was attributed to the non-uniform prevalence of both Ohmic and non-Ohmic shunt pathways. Additionally, device parameters, such as activation energies and barrier heights, may be determined using the model. Future work will also focus on the merits of applying the model in a general case to other thin-film photovoltaic technologies such as $\mathrm{CdTe}$ and $\mathrm{Cu}_{2} \mathrm{ZnSn}(\mathrm{S}, \mathrm{Se})_{4}$.

\section{ACKNOWLEDGEMENTS}

The authors acknowledge J.J.L.M. Meulendijks, C.O. van Bommel, and J.J.A. Zeebregts for their technical support. CIGS device samples were provided from TNO's CIGS baseline process, which is described in the experimental section of the manuscript. This work was financially supported by Interreg Vlaanderen Nederland, Solar flare under project number IVA-VLANED-1.59. The research of M.C. has been funded by the Netherlands Organization for Scientific Research (NWO, Aspasia programma).

\section{REFERENCES}

1. http://www.nrel.gov/ncpv/images/efficiency_chart.jpg, Accessed on 28/12/14.

2. Tessema MM, Giolando DM. Solar Energy Materials and Solar Cells 2012; 107: 9-12.

3. Bosio A, Romeo N, Mazzamuto S, Canevari V. Progress in Crystal Growth and Characterization of Materials 2006; 52: 247-279. 
4. Kaminski A, Marchand JJ, ElOmari H, Laugier A, Le QN, Sarti D. Conduction Processes in Silicon Solar Cells. In Conference Record of the 25th IEEE proceedings 1996 p57 1996; 573-576.

5. Chan DSH, Phang JCH. Ieee Transactions on Electron Devices 1987; 34: 286-293.

6. Clayton AJ, Irvine SJC, Jones EW, Kartopu G, Barrioz V, Brooks WSM. Solar Energy Materials and Solar Cells 2012; 101: 68-72.

7. Dongaonkar S, Servaites JD, Ford GM, Loser S, Moore J, Gelfand RM, Mohseni H, Hillhouse HW, Agrawal R, Ratner MA, Marks TJ, Lundstrom MS, Alam MA. Journal of Applied Physics 2010; 108: 124509-1-124509-10.

8. Rose A. Physical Review 1955; 97: 1538-1544.

9. Williams BL, Major JD, Bowen L, Phillips L, Zoppi G, Forbes I, Durose K. Solar Energy Materials and Solar Cells 2014; 124: 31-38.

10. Williams BL, Taylor AA, Mendis BG, Phillips L, Bowen L, Major JD, Durose K. Applied Physics Letters 2014; 104: 053907-1-053907-5.

11. Kirchartz T, Deledalle F, Tuladhar PS, Durrant JR, Nelson J. Journal of Physical Chemistry Letters 2013; 4: 2371-2376.

12. McIntosh KR, Honsberg CB. The Influence of Edge Recombination on a Solar Cell's Iv Curve. in 16th European Photovoltaic Solar Energy Conference. 2000.
13. Sze SM. Physics of Semiconductor Devices. 2nd Edition, Wiley: New Jersey, 1982.

14. Matsuura H, Okuno T, Okushi H, Tanaka K. Journal of Applied Physics 1984; 55: 1012-1019.

15. Babkair SS, Ansari AA, Al-Twarqi NM. Materials Chemistry and Physics 2011; 127: 296-299.

16. Morsli M, Cattin L, Bernède JC, Kumar P, Chand S. Journal of Physics D: Applied Physics 2010; 43: 335103-1-335103-7.

17. Marsal LF, Pallarès J, Correig X, Calderer J, Alcubilla R. Semiconductor Science and Technology 1996; 11: 1209-1213.

18. Batzner DL, Oszan ME, Bonnet D, Bucher K. Thin Solid Films 2000; 361: 288-292.

19. Stollwerck G, Sites JR. in 13th European PVSEC. 1995. Nice, France.

20. Gloeckler M, Jenkins C, Sites J. in MRS Proceedings 763: 2003.

21. Sharma K, Williams BL, Mittal A, Knoops HCM, Kniknie BJ, Bakker NJ, Kessels WMM, Schropp R, Creatore M. Journal of Photoenergy in press, 2014.

\section{SUPPORTING INFORMATION}

Supporting information may be found in the online version of this article. 